\title{
Dyspnoea severity and pneumonia as predictors of in-hospital mortality and early readmission in acute exacerbations of COPD
}

\author{
J Steer, ${ }^{1}$ E M Norman, ${ }^{1}$ O A Afolabi, ${ }^{1}$ G J Gibson, ${ }^{2}$ S C Bourke ${ }^{1,2}$
}

'Department of Respiratory Medicine, North Tyneside General Hospital, Rake Lane, North Shields, UK

Institute of Cellular Medicine, Newcastle University, Newcastle-upon-Tyne, UK

\section{Correspondence to} Dr John Steer, North Tyneside General Hospital, Northumbria Health NHS Foundation Trust, Rake Lane, North Shields, Tyne and Wear NE29 8NH, UK; john.steer@nhct.nhs.uk

Received 12 April 2011 Accepted 11 August 2011 Published Online First 6 September 2011

\begin{abstract}
Background Rates of mortality and readmission are high in patients hospitalised with acute exacerbations of chronic obstructive pulmonary disease (AECOPD). In this population, the prognostic value of the Medical Research Council Dyspnoea Scale (MRCD) is uncertain, and an extended MRCD (eMRCD) scale has been proposed to improve its utility. Coexistent pneumonia is common and, although the CURB-65 prediction tool is used, its discriminatory value has not been reported.

Methods Clinical and demographic data were collected on consecutive patients hospitalised with AECOPD. The relationship of stable-state dyspnoea severity to inhospital mortality and 28-day readmission was assessed. The discriminatory value of CURB-65, MRCD and eMRCD, in the prediction of in-hospital mortality, was assessed and compared.

Results 920 patients were recruited. $10.4 \%$ died inhospital and $19.1 \%$ of the 824 survivors were readmitted within 28 days of discharge. During their stable state prior to admission, $34.2 \%$ of patients were too breathless to leave the house. Mortality was significantly higher in pneumonic than in non-pneumonic exacerbations $(20.1 \%$ vs $5.8 \%, p<0.001)$. eMRCD was a significantly better discriminator than either CURB-65 or the traditional MRCD scale for predicting in-hospital mortality, and was a stronger prognostic tool than CURB65 in the subgroup of patients with pneumonic AECOPD. Conclusions The severity of dyspnoea in the stable state predicts important clinical outcomes in patients hospitalised with AECOPD. The eMRCD scale identifies a subgroup of patients at a particularly high risk of inhospital mortality and is a better predictor of mortality risk than CURB-65 in exacerbations complicated by pneumonia.
\end{abstract}

\section{BACKGROUND}

Acute exacerbations of chronic obstructive pulmonary disease (AECOPD) requiring hospitalisation are responsible for a substantial proportion of the morbidity and mortality associated with the disease. The 2008 National UK COPD audit showed that $7.7 \%$ of patients hospitalised with exacerbations died during admission and about onethird of those discharged from hospital were readmitted within 90 days. ${ }^{1}$ As disease severity worsens, the frequency of hospital admission increases, ${ }^{2}$ and hospitalisation provides a potential window to identify and intervene in patients at risk of adverse outcome. The ability to accurately risk

\section{Key messages}

What is the key question?

- What is the impact of stable-state dyspnoea and coexistent pneumonia on outcome in patients hospitalised with acute exacerbations of COPD?

\section{What is the bottom line?}

- A novel extended version of the Medical Research Council Dyspnoea Scale (eMRCD) and the presence of pneumonia are both strongly associated with mortality and readmission; in patients with pneumonic exacerbations, eMRCD is a stronger predictor of mortality than CURB-65.

\section{Why read on?}

- These findings should help clinicians identify patients at high risk of death who may benefit from either early escalation of care or early discussion of end-of-life care.

stratify patients presenting to hospital with exacerbations would help to prevent unnecessary admission in those deemed low risk and facilitate earlier intervention to reduce morbidity and mortality in those at high risk.

COPD exacerbations are commonly complicated by radiographic consolidation. ${ }^{3}$ There is debate over whether people with AECOPD and coexistent pneumonia should be included in the definition of AECOPD, with varying practice in the UK. However, pulmonary consolidation was not an exclusion in the major UK national audits of COPD exacerbations ${ }^{1}$ and non-invasive ventilation (NIV), ${ }^{4}$ in which patients with coexistent radiographic consolidation comprised $16 \%$ and $34.2 \%$ respectively. Furthermore, plain chest radiography is relatively insensitive for identifying pulmonary consolidation, with a number of patients with initially negative radiography found to have consolidation on subsequent more detailed imaging. ${ }^{5}$ In addition, Lieberman et al ${ }^{3}$ showed that, compared with patients with non-pneumonic exacerbations, those with coexistent radiographic consolidation were similar in terms of sociodemographic details and severity of the underlying COPD, although they had more abnormal markers of acute clinical and physiological derangement, suggesting that coexistent consolidation identifies patients with a more severe acute illness, but does 
not signify a different disease process. For these reasons we elected to include patients with coexistent consolidation in the present study.

In community-acquired pneumonia the CURB- 65 prognostic tool $^{7}$ is effective and widely used as a guide to clinical management, but its utility in patients with pneumoniacomplicating AECOPD (pAECOPD) is less clear. CURB-65 is also sometimes used to guide clinical decisions in non-pneumonic AECOPD (npAECOPD) and a single prospective study supports this. ${ }^{8}$

In stable COPD, the severity of dyspnoea, measured by the Medical Research Council Dyspnoea Score (MRCD), is a stronger predictor of mortality than forced expiratory volume in $1 \mathrm{~s}\left(\mathrm{FEV}_{1}\right)$ alone. ${ }^{9}$ Its prognostic value in $\mathrm{AECOPD}$ requiring hospitalisation has, however, been infrequently studied, with single reports suggesting that higher MRCD scores are predictive of in-hospital ${ }^{10}$ and 3-year mortality. ${ }^{11} \mathrm{~A}$ small retrospective study from our department described a novel extended version of the MRCD scale (the extended MRC Dyspnoea Score, eMRCD), which had better discrimination than the traditional MRCD scale for the identification of patients at risk of frequent hospital admission, ${ }^{12}$ but this, and the relationship between eMRCD and in-hospital mortality, have not been further investigated.

We have undertaken a prospective observational study in a large population of patients with AECOPD, to compare the predictive ability of both the MRCD and eMRCD scores in relation to both in-hospital mortality and early rehospitalisation. We also assessed the impact of complicating pneumonia on outcome and the utility of CURB-65 in this population.

\section{Methods}

As part of a large prospective observational study, consecutive patients admitted with a primary diagnosis of AECOPD to one of two neighbouring acute general hospitals between December 2008 and June 2010 were recruited. Patients were identified primarily by screening the records of patients admitted with a clinical diagnosis of AECOPD on a daily basis. To ensure maximal data capture, hospital coding records were reviewed in order to identify patients who died or were rapidly discharged prior to identification by the research team, and data were gathered on all eligible patients not previously identified.

Inclusion criteria were admission with AECOPD; a clinical diagnosis of COPD supported by spirometric evidence of airflow obstruction $\left(\mathrm{FEV}_{1} /\right.$ forced vital capacity $\left.<0.70\right)$ during a period of clinical stability; age at least 35 years; smoking history of at least 10 cigarette pack years; and admission from the primary place of residence. Patients were not eligible for inclusion if they had previously been included in the study; they were being treated with domiciliary ventilation; they had life-threatening comorbidity that was expected to limit their life to less than 12 months; or if they had a primary admission diagnosis other than AECOPD.

Sociodemographic details, $\mathrm{FEV}_{1}$ and body mass index (BMI) on admission were recorded. Only $\mathrm{FEV}_{1}$ results obtained within 2 years of admission were used in data analysis. During their hospital stay, patients were asked to report their level of dyspnoea during their stable state in the preceding 3 months. The eMRCD $^{12}$ categorises patients with traditional MRCD scores of 5 as either $5 \mathrm{a}$ or $5 \mathrm{~b}$ depending on their ability to manage personal care (washing and dressing). This was documented routinely and recorded along with the traditional MRCD score (table 1). The presence of consolidation on the admission chest radiograph, as documented by the admitting medical team, and
Table 1 The traditional Medical Research Council Dyspnoea Scale (MRCD) $^{13}$ and the extended version (eMRCD) ${ }^{12}$

\begin{tabular}{lll}
\hline Limitation due to breathlessness & MRCD & eMRCD \\
\hline $\begin{array}{l}\text { Breathless only with strenuous exercise } \\
\text { Breathless when hurrying on the level or } \\
\text { walking up a slight hill }\end{array}$ & 1 & 2 \\
$\begin{array}{l}\text { Walks slower than peers, or stops when } \\
\text { walking on the flat at own pace }\end{array}$ & 3 & \\
$\begin{array}{l}\text { Stops after walking } 100 \mathrm{~m} \text {, or for a few } \\
\text { minutes, on the level }\end{array}$ & 4 \\
$\begin{array}{l}\text { Too breathless to leave the house } \\
\text { \& independent in washing and/or dressing }\end{array}$ & 5 & $\mathbf{5 a}$ \\
$\mathbf{8}$ dependent in washing and dressing & & $\mathbf{5 b}$ \\
\hline
\end{tabular}

the CURB-65 score were recorded. The admission was labelled as pAECOPD or npAECOPD depending on whether consolidation was present or absent. Length of stay, need for ventilatory support (non-invasive or invasive ventilation), in-hospital and 30-day mortality, and readmission to hospital within 28 and 90 days of discharge were gathered by reviewing the hospital records. The local NHS Research Ethics Committee approved the project and advised that formal consent from individual patients was not required.

\section{Statistical analysis}

For variables with missing data, cases were excluded analysis by analysis. Descriptive statistics were used to characterise the patient sample, using proportions, means with standard deviations, or medians with inter-quartile ranges (IORs) if appropriate. Bivariate comparisons were performed for pneumonic and non-pneumonic AECOPD using the $\chi^{2}$ test (gender and need for assisted ventilation), Student $t$ test (age, FEV $1 \%$ predicted and BMI) and Mann-Whitney $U$ test (MRCD and length of stay). Differences in outcome between eMRCD 5a and $5 \mathrm{~b}$, and between pAECOPD and npAECOPD, were assessed using the $\chi^{2}$ test. The discriminatory value of MRCD, eMRCD and CURB-65 in relation to in-hospital mortality was determined by computing the area under the ROC curve (AUROC) with 95\% CIs. AUROC was compared between MRCD, eMRCD and CURB-65 using the method of DeLong et al ${ }^{14}$ and was computed using SigmaPlot version 11. All other statistical analyses were performed using SPSS V.15.0 for Windows and a two-sided $p$ value $<0.05$ was taken as statistically significant.

\section{RESULTS}

Data were obtained from 920 patients. Data capture was complete for all variables apart from $\mathrm{FEV}_{1}$ within 2 years of admission (14.3\% missing) and BMI on admission (4.3\% missing). The mean age of the patients was 73.1 years (SD 10.0) and $53.9 \%$ were women (table 2). Most patients had severe airflow obstruction (mean $\mathrm{FEV}_{1} \%$ predicted 44.1, SD 18.0). Mean BMI was normal $\left(24.6 \mathrm{~kg} / \mathrm{m}^{2}\right.$, SD 6.4) and approximately one-third of patients had radiographic evidence of pulmonary consolidation. Compared with patients with no consolidation, they were older, had slightly better preserved $\mathrm{FEV}_{1}$, and were more likely to have required assisted ventilation. Almost all $(98.0 \%)$ patients receiving assisted ventilation were initially treated with NIV, with only four being immediately intubated and ventilated. Of patients in whom NIV failed, only four further patients progressed to invasive ventilation. Of the 51 patients with severe dyspnoea (eMRCD 5b) who met the criteria for assisted ventilation $(\mathrm{pH}<7.35$ and arterial carbon dioxide pressure $>6 \mathrm{kPa}$ ), 44 received it and seven were not ventilated, but instead received end-of-life care. 
Table 2 Population characteristics

\begin{tabular}{|c|c|c|c|c|c|c|c|c|}
\hline & $\mathbf{N}$ & $\begin{array}{l}\text { Women, } \\
\text { n (\%) }\end{array}$ & $\begin{array}{l}\text { Mean age, } \\
\text { years (SD) }\end{array}$ & $\begin{array}{l}\text { Mean FEV }{ }_{1} \% \\
\text { predicted (SD)* }\end{array}$ & $\begin{array}{l}\text { Mean BMI } \\
\text { (SD) } \dagger\end{array}$ & $\begin{array}{l}\text { Median MRCD } \\
\text { (IQR) }\end{array}$ & $\begin{array}{l}\text { Assisted ventilation, } \\
\text { n }(\%) \neq\end{array}$ & $\begin{array}{l}\text { In-hospital deaths, } \\
\text { n (\%) }\end{array}$ \\
\hline Total population & 920 & $496(53.9)$ & $73.1(10.0)$ & $44.1(18.0)$ & $24.6(6.4)$ & $4(4-5)$ & $199(21.6)$ & $96(10.4)$ \\
\hline npAECOPD & 621 & $348(56)$ & $71.7(10.2) \S$ & $43.2(18.0)^{* *}$ & $24.7(6.5)$ & $4(3-5)$ & $118(19.0) 9$ & $36(5.8) \S$ \\
\hline
\end{tabular}

*If performed within 2 years of admission, $n=788$.

$\dagger \mathrm{n}=880$.

$\neq$ Received either non-invasive ventilation or invasive ventilation during hospital admission

Significant difference between pneumonic and non-pneumonic AECOPD: $\S p<0.001, \uparrow p=0.0053,{ }^{* *} p=0.026$

$\mathrm{BMI}$, body mass index; $\mathrm{FEV}_{1}$, forced expiratory volume in $1 \mathrm{~s}$; IQR, interquartile range; MRCD, Medical Research Council Dyspnoea Scale; npAECOPD, non-pneumonic acute exacerbations of chronic obstructive pulmonary disease; pAECOPD, pneumonic acute exacerbations of chronic obstructive pulmonary disease.

Ninety-six patients $(10.4 \%)$ died during admission (86 from respiratory disease and 10 from non-respiratory disease) and 115 $(12.5 \%)$ died within 30 day of admission (table 3$)$. Of the 824 surviving to discharge, $157(19.1 \%)$ were readmitted to hospital within 28 days and $16(1.9 \%)$ died within 28 days of discharge without being rehospitalised. Overall, 275 patients (33.4\%) were readmitted to hospital within 90 days of discharge (table 4). Median length of stay was 6 days (IOR 3-11) and pAECOPD was associated with a longer hospital stay than npAECOPD ( 7 days vs 6 days; $p<0.001$ ). The in-hospital mortality rate for npAECOPD was 5.8\% (36/621), whereas 20.1\% (60/299) with pAECOPD died in-hospital $(p<0.001)$. Twenty-eight-day readmission rates of those initially presenting with pneumonic and non-pneumonic AECOPD were similar $(19.5 \%$ and $18.0 \%$ respectively; $\mathrm{p}=0.62$ ).

The distribution of patients within each dyspnoea grade and the frequency of outcomes for the total population and the pAECOPD and npAECOPD subgroups are shown in tables 3 and 4. Pre-admission, during a period of clinical stability, 315 (34.2\%) patients were too breathless to leave the house (MRCD 5). Of these, $173(54.9 \%)$ were independent in washing and dressing (eMRCD 5a) and 142 (45.1\%) were dependent in washing and dressing (eMRCD 5b).

Of the 96 patients who died in hospital, 30 were eMRCD 5a (17.3\% mortality) and 47 eMRCD $5 b$ ( $33.1 \%$ mortality) $(\mathrm{p}=0.0012)$. In the npAECOPD group, patients with eMRCD $5 \mathrm{~b}$ had a significantly higher in-hospital mortality rate than those with eMRCD $5 \mathrm{a}(\mathrm{p}=0.048)$; in the pAECOPD group, there was a similar but non-significant association $(\mathrm{p}=0.069)$ (table 3 ).

The 28-day readmission rate was significantly higher for those with eMRCD $5 b$ than those with eMRCD $5 a$ for the total population $(p=0.044)$ and for the subgroup with npAECOPD $(\mathrm{p}=0.0005)$ (table 4). In $\mathrm{pAECOPD}$, the rate was non-significantly lower with eMRCD $5 b$ than eMRCD $5 a(p=0.13)$; this is

Table 3 Relation of dyspnoea grade and presence or absence of consolidation to mortality

\begin{tabular}{|c|c|c|c|c|c|}
\hline \multirow{2}{*}{$\begin{array}{l}\text { Dyspnoea } \\
\text { grade }\end{array}$} & \multirow[b]{2}{*}{$\mathbf{n}$} & \multicolumn{3}{|c|}{ In-hospital mortality, \% } & \multirow{2}{*}{$\begin{array}{l}\text { 30-day } \\
\text { mortality, \%* } \\
\text { Total }\end{array}$} \\
\hline & & Total & npAECOPD & pAECOPD & \\
\hline 1 & 6 & 0 & 0 & 0 & 0 \\
\hline 2 & 46 & 0 & 0 & 0 & 0 \\
\hline 3 & 171 & 2.3 & 1.6 & 4.4 & 2.9 \\
\hline 4 & 382 & 3.9 & 0.4 & 11.8 & 6.0 \\
\hline 5 & 315 & 24.4 & 16.6 & 37.9 & 27.6 \\
\hline $5 a$ & 173 & $17.3 \dagger$ & $12.4 \ddagger$ & 28.8 & $22.5 \neq$ \\
\hline $5 b$ & 142 & $33.1 \dagger$ & $23.1 \neq$ & 45.3 & $33.8 \neq$ \\
\hline
\end{tabular}

*From time of hospital admission.

†Significant difference between $5 a$ and $5 b, p<0.01$

$\neq$ Significant difference between $5 a$ and $5 b, p<0.05$.

npAECOPD, non-pneumonic acute exacerbations of chronic obstructive pulmonary disease;

pAECOPD, pneumonic acute exacerbations of chronic obstructive pulmonary disease. likely to be due to a survivor effect given the high in-hospital mortality in the former group. Of the 16 patients who died within 28 days of discharge without being readmitted to hospital, three were eMRCD 3-4, eight were eMRCD $5 \mathrm{a}$ and five were eMRCD $5 b$ (eMRCD $5 a$ vs eMRCD $5 b$ : $p=0.91$ ).

Of the 299 patients with pAECOPD, median CURB-65 score was 2 (IOR 1-3) and 109 (36.5\%) had CURB-65 scores of 3-5 and therefore a high risk of mortality (table 5). Mortality rates for each CURB-65 score were higher in pAECOPD than npAECOPD.

The discriminatory ability of MRCD, eMRCD and CURB-65 to predict in-hospital mortality was assessed and compared using areas under receiver operating characteristic curves (AUROC curves) (table 6). In the population as a whole, eMRCD had significantly better discrimination for in-hospital mortality than either MRCD $(p=0.0012)$ or CURB-65 $(p=0.019)$, and in the npAECOPD group there was a nonsignificant trend to better discrimination for eMRCD compared with both CURB-65 $(p=0.053)$ and MRCD $(p=0.057)$. In pAECOPD, eMRCD performed significantly better than CURB65 for both in-hospital (AUROC=0.759 vs $0.661, p=0.017$ ) and 30-day (AUROC $=0.753$ vs $0.640, p=0.040$ ) mortality. The discriminative strength of eMRCD for in-hospital mortality is shown in figure 1.

\section{DISCUSSION}

Of our population of patients admitted with AECOPD, more than one-third were housebound (MRCD 5) in the stable state. We found that the severity of dyspnoea in the stable state was strongly associated with both in-hospital mortality and early readmission. We have also shown that extending the traditional MRCD scale to take account of a person's ability to manage personal care (eMRCD) improves the prognostic strength of the instrument for both in-hospital mortality

Table 4 Relation of dyspnoea grade and presence or absence of consolidation to readmission

\begin{tabular}{|c|c|c|c|c|c|}
\hline \multirow{2}{*}{$\begin{array}{l}\text { Dyspnoea } \\
\text { grade }\end{array}$} & \multirow[b]{2}{*}{$\mathbf{n}$} & \multicolumn{3}{|c|}{ 28-day readmission, \%* } & \multirow{2}{*}{$\begin{array}{l}\text { 90-day } \\
\text { readmission, \%* } \\
\text { Total }\end{array}$} \\
\hline & & Total & npAECOPD & $\overline{\text { pAECOPD }}$ & \\
\hline 1 & 6 & 0 & 0 & 0 & 16.7 \\
\hline 2 & 46 & 6.5 & 10.7 & 0 & 10.9 \\
\hline 3 & 167 & 12.0 & 11.3 & 14.0 & 19.2 \\
\hline 4 & 367 & 19.9 & 20.6 & 18.1 & 34.1 \\
\hline 5 & 238 & 25.6 & 25.9 & 25.0 & 47.1 \\
\hline $5 a$ & 143 & $21.0 \dagger$ & $17.0 \neq$ & 32.4 & $39.9 \neq$ \\
\hline $5 b$ & 95 & $32.6 \dagger$ & $41.7 \ddagger$ & 17.1 & $57.9 \ddagger$ \\
\hline
\end{tabular}

*Of patients surviving to discharge, $\mathrm{n}=824$.

† Significant difference between $5 a$ and $5 b, p<0.05$

$\neq$ Significant difference between $5 a$ and $5 b, p<0.01$

npAECOPD, non-pneumonic acute exacerbations of chronic obstructive pulmonary disease; PAECOPD, pneumonic acute exacerbations of chronic obstructive pulmonary disease. 
Table 5 Distribution of patients, and rates of mortality, according to CURB-65 score

\begin{tabular}{|c|c|c|c|c|c|c|}
\hline \multirow{2}{*}{$\begin{array}{l}\text { CURB-65 } \\
\text { score }\end{array}$} & \multicolumn{2}{|c|}{ Total population } & \multicolumn{2}{|c|}{ npAECOPD } & \multicolumn{2}{|c|}{ pAECOPD } \\
\hline & $\overline{n(\%)}$ & Mortality \% & $\bar{n}$ & Mortality \% & $\bar{n}$ & Mortality \% \\
\hline 0 & 135 (14.7) & 4.4 & 115 & 2.6 & 20 & 15 \\
\hline 1 & $278(30.2)$ & 4.0 & 208 & 1.9 & 70 & 10.0 \\
\hline 2 & $295(32.1)$ & 9.5 & 195 & 6.2 & 100 & 16 \\
\hline 3 & $169(18.4)$ & 20.1 & 87 & 16.1 & 82 & 24.4 \\
\hline 4 & $36(3.9)$ & 36.1 & 15 & 20 & 21 & 47.6 \\
\hline 5 & $7(0.8)$ & 57.1 & 1 & 0 & 6 & 66.7 \\
\hline
\end{tabular}

npAECOPD, non-pneumonic acute exacerbations of chronic obstructive pulmonary disease $\mathrm{pAECOPD}$, pneumonic acute exacerbations of chronic obstructive pulmonary disease.

and early rehospitalisation following discharge, and identifies a subgroup of patients at particularly high risk of in-hospital mortality (33.1\% with eMRCD $5 b$ ). In addition, our results highlight the adverse prognostic effect of radiographic pulmonary consolidation complicating AECOPD (in-hospital mortality of $20.1 \%$ in the pAECOPD group compared with $5.8 \%$ in the npAECOPD group).

Our study has notable strengths. Great effort was made to ensure inclusion of, and complete data capture in, all patients hospitalised with AECOPD during the period of the study, including those in whom the diagnosis of COPD had not previously been made and patients with a very short length of stay who otherwise might not have been brought to our attention. Our study also has the major strength of a large population of patients with AECOPD admitted consecutively to one of two large general hospitals. Our entry criteria were developed to make the study as inclusive as possible, and to reflect the general population of patients hospitalised with AECOPD in the UK. We are also aware of certain limitations to the study. In $15 \%$ of patients no spirometric measurements were available within 2 years of admission; spirometric support for the diagnosis of COPD was, however, obtained for all these patients. Although clinical information was obtained by a number of nursing and medical staff, standard protocols for gathering and recording the data were in place for patients admitted with AECOPD. Finally, the presence or absence of radiographic consolidation was recorded by the admitting medical team and radiographs were not specifically reviewed for the purpose of the study.

In comparison to the 2008 UK National COPD Audit, ${ }^{1}$ the overall in-hospital mortality in our study is somewhat higher; this is attributable mainly to the higher prevalence of coexistent consolidation in our cohort $32.5 \%$ compared with $16 \%$ in the National COPD Audit) and the associated worse prognosis. Different centres vary as to whether they include cases with pneumonia; to allow valid comparison of relative mortality, we suggest future audits stratify outcome according to the presence of coexistent consolidation. The readmission rate at 90 days

Table 6 Area under receiver operating curve for prediction of in-hospital mortality

\begin{tabular}{llll}
\hline & MRCD & eMRCD & CURB-65 \\
\hline Total & $0.769(0.73-0.81)$ & $0.794(0.75-0.84)^{*} \dagger$ & $0.717(0.66-0.77)$ \\
npAECOPD & $0.809(0.75-0.87)$ & $0.833(0.77-0.90)$ & $0.719(0.63-0.81)$ \\
pAECOPD & $0.740(0.68-0.80)$ & $0.759(0.70-0.82) \dagger$ & $0.661(0.58-0.74)$ \\
\hline
\end{tabular}

*Significant difference compared with MRCD, $\mathrm{p}<0.01$

†Significant difference compared with CURB-65, $p<0.05$.

eMRCD, extended Medical Research Council Dyspnoea Scale; MRCD, Medical Research

Council Dyspnoea Scale; npAECOPD, non-pneumonic acute exacerbations of chronic

obstructive pulmonary disease; $\mathrm{PAECOPD}$, pneumonic acute exacerbations of chronic

obstructive pulmonary disease.

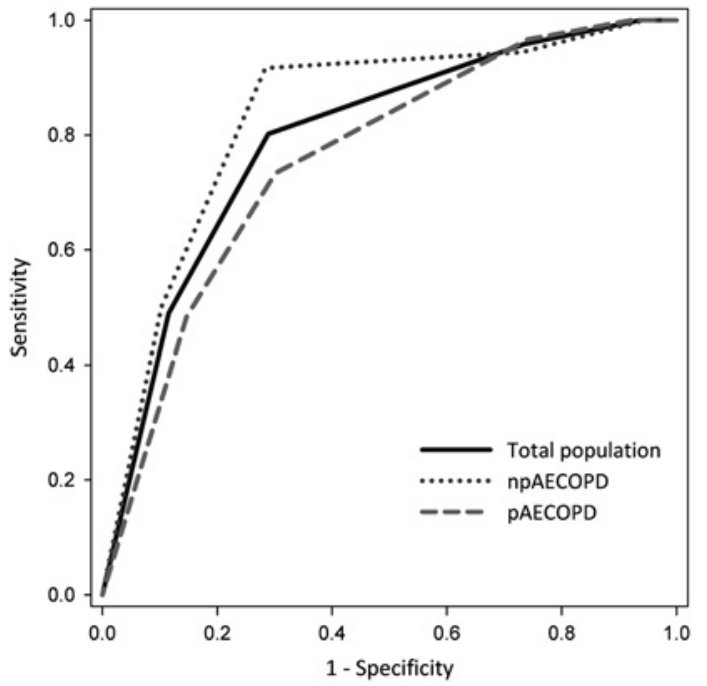

Figure 1 The discrimination of eMRCD for in-hospital mortality for the total population, non-pneumonic and pneumonic exacerbations of COPD.

$(33.4 \%)$ is almost identical to that reported in the National COPD Audit. ${ }^{1}$

The severity of stable-state dyspnoea in patients hospitalised with AECOPD has rarely been reported previously. Similarly to our finding, the 2008 UK National COPD Audit ${ }^{1}$ suggested that approximately $30 \%$ of admitted patients were too breathless to leave the house (MRCD 5), but the conclusion was limited by missing data in more than half of the patients audited. Other studies ${ }^{11} 1516$ have recorded dyspnoea severity only in patients surviving to discharge, which underestimates its importance because of its strong association with mortality. Higher MRCD scores have previously been shown to be associated with greater in-hospital mortality in patients attending the emergency department with AECOPD, ${ }^{10}$ an association we have confirmed for all patients hospitalised with AECOPD. A recent study ${ }^{17}$ showed an association between the traditional MRCD score and hospital readmission in patients enrolled in an early supported discharge scheme, but to our knowledge a similar association between MRCD and hospital readmission in all patients hospitalised with AECOPD has not been reported.

Greater functional dependence has been shown independently to predict hospital readmission, ${ }^{15}{ }^{18}$ and performance status, which includes an assessment of a patient's ability to self care, has been shown to be predictive of 3-month mortality following admission. ${ }^{19}$ Also, in patients surviving to discharge, a high level of functional dependence is associated with long-term mortality. ${ }^{162021}$ Most of the deaths (80\%) in our study occurred in patients with severe stable-state dyspnoea (MRCD 5). We have shown that combining a measure of functional dependence with the assessment of dyspnoea severity (eMRCD) improves the predictive ability of the traditional MRCD scale, with a significantly higher risk of mortality in patients housebound and dependent in washing and dressing (eMRCD 5b) than in those housebound but independent in washing and/or dressing (eMRCD 5a).

Clinical decisions were in the hands of the admitting medical teams and uninfluenced by our study; however, we recognise that severe disability is likely to have been an important consideration in determining the management of individual patients. However, our finding does not appear to be explained by early introduction of palliative care, or limiting the level of care, in this population because even among patients with the 
most severe limitation (eMRCD 5b), most of those potentially eligible for assisted ventilation received it, and there was no difference in this regard between eMRCD $5 a$ and eMRCD $5 b$.

Using the extended scale, each increase in dyspnoea severity was accompanied by a significantly higher mortality, and the prediction of in-hospital mortality was significantly better using eMRCD than MRCD (AUROC=0.794 vs 0.769; $\mathrm{p}=0.0012$ ) (table 6). For the total population and for the npAECOPD group, eMRCD also improved identification of patients at risk of hospital readmission (table 4).

Our study shows that co-existent consolidation is common in patients with AECOPD and is associated with a high mortality rate. In community-acquired pneumonia, 30-day mortality rates according to CURB-65 have been previously reported to be: CURB-65 $0-1=1.5 \%$; CURB-65 2=9.2\%; and CURB-65 3-5=22.4\%. ${ }^{7}$ Our study shows that the combination of AECOPD and pneumonia is associated with higher mortality rates than pneumonia alone: pAECOPD and CURB-65 0-1=11.1\% in-hospital mortality; pAECOPD and CURB-65 2=16\%; and pAECOPD and CURB-65 3-5=31.2\%. Therefore, although the CURB-65 risk stratification tool is frequently used to guide management in $\mathrm{PAECOPD}$, our data show that it underestimates the risk of mortality in this population. The results of AUROC analysis (table 6) confirm that in PAECOPD, the performance of CURB-65 was only moderate (AUROC $=0.661$ ), and eMRCD (AUROC $=0.759$ ) was a significantly better discriminator for both in-hospital $(p=0.017)$ and 30-day mortality $(p=0.040)$. Our results concur with a recent study ${ }^{8}$ that showed CURB-65 to have good discrimination for in-hospital mortality in nPAECOPD (AUROC=0.733), but in our study, the eMRCD outperformed CURB-65 for all patients and we suggest that it is the better predictive tool.

Previous studies to identify prognostic indices in AECOPD requiring hospitalisation have been performed infrequently and (with the exception of patients requiring intensive care) robust clinical tools to aid management of such patients have not been developed. ${ }^{22}$ The present study shows that the assessment of dyspnoea severity in patients hospitalised with AECOPD is simple to perform, is a strong predictor of outcome and may therefore provide valuable information which could influence management. For example, of the total population, no patients hospitalised with eMRCD grade 1 or 2 died either in hospital or at home shortly after discharge, and in the npAECOPD group with eMRCD 1-4, in-hospital mortality was very low and therefore many of these patients might have been safely managed in the community. Alternatively, one-third of all patients with eMRCD $5 \mathrm{~b}$, and almost a half of patients with coexistent pneumonia and eMRCD $5 b$, did not survive to discharge and such patients might benefit from either early escalation of care or, in some cases, early discussion of end-of-life care.

We conclude that severe dyspnoea is common in patients hospitalised with AECOPD and the severity of dyspnoea is a strong predictor of mortality and early rehospitalisation. A novel extension of the traditional scale, the eMRCD, improves its predictive ability for both mortality and readmission. Pneumonic exacerbations of COPD are an important and common clinical problem with overall worse outcome than primary community acquired pneumonia. The CURB-65 prediction tool for pneumonia performs less well in patients with pAECOPD. Further research is needed to develop practical prognostic tools in AECOPD in order to aid clinical management.
Correction notice This article has been corrected since it was published Online First Tables 3 and 4 contained numbers which were not aligned in the columns correctly.

\section{Competing interests None.}

Ethics approval This study was conducted with the approval of NHS County Durham and Tees Valley 1 Research Ethics Committee.

Contributors SCB designed and obtained funding for the study. Data collection was performed by JS and EN. The original eMRCD scale was developed by GA and EN. Data analysis was performed by JS, and supervised by both GJG and SCB. The manuscript was drafted by JS; GA, GJG and SCB helped with its revision. GJG and SCB provided JS with ongoing educational supervision.

Provenance and peer review Not commissioned; externally peer reviewed.

\section{REFERENCES}

1. Royal College of Physicians, British Thoracic Society, British Lung Foundation. Report of the National Chronic Obstructive Pulmonary Disease Audit 2008: Clinical audit of COPD exacerbations admitted to acute NHS trusts across the UK London: Royal College of Physicians, 2008.

2. Anzueto A, Leimer I, Kesten S. Impact of frequency of COPD exacerbations on pulmonary function, health status and clinical outcomes. Int J Chron Obstruct Pulmon Dis 2009:4:245-51.

3. Lieberman D, Lieberman $D$, Gelfer $Y$, et al. Pneumonic vs nonpneumonic acute exacerbations of COPD. Chest 2002;122:1264-70.

4. Davidson C. 2010 Adult Non-Invasive Ventilation Audit summary report. British Thoracic Society, London, 2011.

5. Syrjala H, Broas M, Suramo I, et al. High-resolution computed tomography for the diagnosis of community-acquired pneumonia. Clin Infect Dis 1998;27:358-63.

6. Hagaman JT, Rouan GW, Shipley RT, et al. Admission chest radiograph lacks sensitivity in the diagnosis of community-acquired pneumonia. Am J Med Sci 2009;337:236-40.

7. Lim WS, van der Eerden MM, Laing $R$, et al. Defining community acquired pneumonia severity on presentation to hospital: an international derivation and validation study. Thorax 2003;58:377-82.

8. Chang CL, Sullivan GD, Karalus N, et al. Predicting early mortality in acute exacerbation of chronic obstructive pulmonary disease using the CURB65 score. Respirology 2011;16:146-51.

9. Nishimura K, Izumi T, Tsukino $\mathbf{M}$, et al. Dyspnea is a better predictor of 5 -year survival than airway obstruction in patients with COPD. Chest 2002;121:1434-40.

10. Roche N, Zureik M, Soussan D, et al. Predictors of outcomes in COPD exacerbation cases presenting to the emergency department. Eur Respir J 2008;32:953-61.

11. Tsimogianni AM, Papiris SA, Stathopoulos GT, et al. Predictors of outcome after exacerbation of chronic obstructive pulmonary disease. J Gen Intern Med 2009:24:1043-8.

12. Norman EM, Mylotte A, Watson P, et al. Factors associated with length of stay and re-admissions in patients with chronic obstructive pulmonary disease. Am J Respir Crit Care Med 2001:163:A508.

13. Bestall JC, Paul EA, Garrod R, et al. Usefulness of the Medical Research Council (MRC) Dyspnoea scale as a measure of disability in patients with chronic obstructive pulmonary disease. Thorax 1999:54:581-6.

14. DeLong ER, DeLong DM, Clarke-Pearson DL. Comparing the areas under two or more correlated receiver operating characteristic curves: a nonparametric approach Biometrics 1988;44:837-45.

15. Chu CM, Chan VL, Lin AW, et al. Readmission rates and life threatening events in COPD survivors treated with non-invasive ventilation for acute hypercapnic respiratory failure. Thorax 2004;59:1020-5.

16. Almagro $\mathbf{P}$, Calbo E, Ochoa de Echaguen A, et al. Mortality after hospitalization for COPD. Chest 2002;121:1441-8.

17. Dunican EM, Deering BM, Ryan DM, et al. Factors that predict failure in home management of an acute exacerbation of COPD. Thorax 2011:66:358-9.

18. Lau AC, Yam LY, Poon E. Hospital re-admission in patients with acute exacerbation of chronic obstructive pulmonary disease. Respir Med 2001:95:876-84

19. Roberts CM, Lowe D, Bucknall CE, et al. Clinical audit indicators of outcome following admission to hospital with acute exacerbation of chronic obstructive pulmonary disease. Thorax 2002:57:137-41.

20. Yohannes AM, Baldwin RC, Connolly MJ. Predictors of 1-year mortality in patients discharged from hospital following acute exacerbation of chronic obstructive pulmonary disease. Age Ageing 2005;34:491-6.

21. Connors AF Jr, Dawson NV, Thomas C, et al. Outcomes following acute exacerbation of severe chronic obstructive lung disease. The SUPPORT investigators (Study to Understand Prognoses and Preferences for Outcomes and Risks of Treatments) [erratum appears in Am J Respir Crit Care Med 1997 Jan;155(1):386] Am J Respir Crit Care Med 1996;154:959-67.

22. Steer J, Gibson GJ, Bourke SC. Predicting outcomes following hospitalization for acute exacerbations of COPD. OJM 2010;103:817-29. 